\title{
Caracterización de Klebsiella pneumoniae productora de la $\beta$-lactamasa SHV-5, en una unidad de cuidados intensivos
}

\author{
Verónica Andrade, M en $C_{1}{ }^{(1)}$ Grupo de Resistencia Bacteriana, ${ }^{(2)}$ Jesús Silva, Dr en C.(1)
}

\author{
AndradeV, Grupo de Resistencia Bacteriana, Silva J. \\ Caracterización de Klebsiella pneumoniae productora \\ de la $\beta$-lactamasa SHV-5, en una unidad \\ de cuidados intensivos. \\ Salud Publica Mex 2004;46:524-528. \\ El texto completo en inglés de este artículo está \\ disponible en: http://www.insp.mx/salud/46/eng
}

\section{Resumen}

Objetivo. Caracterizar molecularmente los aislamientos de Klebsiella pneumoniae obtenidos de pacientes pediátricos y del personal de salud en la unidad de cuidados intensivos de un hospital de tercer nivel de atención en la Ciudad de México, D istrito Federal. Material y métodos. Se analizaron 15 aislamientos de Klebsiella pneumoniae colectadas de un brote durante el mes de junio de 1996, ocho de pacientes y siete de personal del Hospital Infantil de México. Los aislamientos fueron caracterizados por electroforesis en gel de campos pulsados (EGCP), amplificación azarosa del polimorfismo de ADN por reacción en cadena de la polimerasa (AAPD-PCR), y serotipificación, isoelectroenfoque de $\beta$-lactamasas y secuenciación nucleotídica de productos de la reacción en cadena de la polimerasa. Resultados. El serotipo predominante fue el 61 y correlacionó con los perfiles deAAPD-PCR y EG CP en 11 de los 15 aislamientos. Se identificó una clona predominante productora de SHV-5 con una alta letalidad. Conclusiones. Las técnicas de biología molecular fueron herramientas muy útiles en la caracterización de la clona de K. pneumoniae identificada en pacientes y el personal hospitalario, que sugirieron una posible transmisión cruzada. Estos resultados ilustran que se debe apoyar el fortalecimiento de los pro-

\author{
Andrade V, Grupo de Resistencia Bacteriana, Silva J. \\ Characterization of SHV-5 $\beta$-lactamase-producing \\ Klebsiella pneumoniae in an Intensive \\ Care Unit. \\ Salud Publica Mex 2004;46:524-528. \\ The English version of this paper \\ is available at: http://www.insp.mx/salud/46/eng
}

\begin{abstract}
A bstract
Objective To perform the molecular characterization of Klebsiella pneumoniae isolates from pediatric patients and health care workers at the intensive care unit of a tertiary care hospital in Mexico C ity. Material and Methods. Fifteen Klebsiella pneumoniae isolates collected during an outbreak in June 1996 were analyzed; eight were from patients and seven from health care workers of Mexico's C hildren's Hospital. Characterization of isolates was carried out by pulsed field gel electrophoresis (PFGE), random amplified polymorphic DN A polymerase chain reaction (RAPD-PCR) and serotyping, $\beta$-Lactamase isoelectric focusing (IEF), and nucleotide sequencing of PCR products. Results Serotype 61 was predominant and correlated with genomic fingerprints of RAPD and PFGE in 11 of 15 isolates. 0 ne SHV-5producer predominant clone with a high case-fatality rate was identified. Conclusions Molecular biology techniques are useful tools to characterize the K. pneumoniae clone isolated from patients and health care workers, suggesting potential cross-transmission. These data call for strengthening control programs to prevent dissemination of nosocomial infections in the studied hospital. The English version of this paper is available at: http://www.insp.mx/ salud/46/eng
\end{abstract}

Este trabajo recibió apoyo del Consejo N acional de Ciencia y Tecnología (1892N -P, 30938-M y 41712-Q).

(1) Instituto $\mathrm{N}$ acional de Salud Pública, C entro de Investigación sobre Enfermedades Infecciosas. Cuernavaca, Morelos, México.

(2) Grupo de Resistencia Bacteriana: Luz Elena Espinosa de los Monteros, Dr en C,Verónica Jiménez, Biol. Hospital Infantil de México Federico Gómez. México, D F, México. Carlos Cervantes, Dr en C. Instituto de Investigaciones Q uímico-Biológicas, U niversidad Michoacana, Morelia, Michoacán, México.

Fecha de recibido: 2 de diciembre de 2003 - Fecha de aprobado: 1 de septiembre de 2004

Solicitud de sobretiros: Dr. Jesús Silva. Departamento de Resistencia Bacteriana. Instituto Nacional de Salud Pública, Centro de Investigación sobre Enfermedades Infecciosas. A venida Universidad 655, colonia Santa María A huacatitlán 62508 Cuernavaca, Morelos, México.

Correo electrónico jsilva@ correo.insp.mx 
gramas de control para evitar la diseminación de infecciones nosocomiales en esa unidad en estudio. El texto completo en inglés de este artículo está disponible en: 46/eng

Palabras clave: Klebsiella pneumoniae; técnica de tipificación bacteriana; electroforesis en gel de campo pulsado; reacción en cadena de la polimerasa; técnica del AD N polimorfo amplificado aleatorio; México
Keyword: Klebsiella pneumoniae; bacterial typing techniques; electro pho resis, gel pulsed-field; polymerase chain reaction; random amplified polymorphic DN A technique; Mexico
K lebsiella pneumoniae es un patógeno oportunista colonizador de piel y mucosas de pacientes hospitalizados que pueden presentar infecciones invasoras como bacteriemias o septicemias. ${ }^{1}$ Usualmente las manos contaminadas del personal son el vehículo responsable de brotes epidémicos. ${ }^{2}$ Las $\beta$-lactamasas de espectro extendido (BLEE) son enzimas derivadas de las familias TEM y SHV, codificadas en plásmidos, que han substituido de 1 a 3 aminoácidos cercanos al sitio activo confiriendo resistencia a aztreonam, cefotaxima y ceftazidima. ${ }^{3}$ En México existen algunos reportes que muestran a K. pneumoniae como uno de los principales organismos causantes de infecciones intrahospitalarias, que causan niveles significativos de morbilidad y mortalidad. ${ }^{4-6}$

En este trabajo se describen las características moleculares de K. pneumoniae productora de BLEE tipo SHV-5, entre pacientes de la unidad de cuidados intensivos neonatal y el personal de un hospital pediátrico de la Ciudad de México.

\section{Material y métodos}

Cepas bacterianas. Se analizaron 15 aislamientos de $K$. pneumoniae colectados de un brote durante el mes de junio de 1996 en la Unidad de Cuidados Intensivos Neonatal (UCIN), procedentes del Hospital Infantil de México. Las características de los aislamientos se muestran en el cuadro I. Las cepas fueron designadas con un número arbitrario, seguido de la letra N (neonato) o P (personal). Los aislamientos fueron identificados con el sistema automatizado MicroScan (DADE, combo 20).

Pruebas de susceptibilidad antimicrobiana y confirmación en la producción de $\beta$-lactamasas de espectro extendido (BLEE). La concentración mínima inhibitoria (CMI) se determinó por el método de dilución en placa, y la prueba confirmatoria para la producción de BLEE se realizó de acuerdo con las guías que describe el Natio- nal Committee for Clinical Laboratory Standards (USA, NCCLS). ${ }^{7}$

Aislamiento de plásmidos y transferencia de la resistencia. La extracción de plásmidos se efectuó por el método de Kieser. ${ }^{8}$ La transferencia de la resistencia a cefotaxima y ampicilina se hizo según el método de Miller $\mathrm{J},{ }^{9}$ usando como cepa receptora a Escherichia coli J53-2 (F-, pro, met, Rif ${ }^{\mathrm{r}}$ ), seleccionando en forma independiente en agar Luria suplementado con rifampicina $(100 \mu \mathrm{g} / \mathrm{ml})$ en combinación con cefotaxima $(1 \mu \mathrm{g} / \mathrm{ml})$ o con ampicilina $(100 \mu \mathrm{g} / \mathrm{ml})$.

Serotipificación. Los aislamientos fueron ensayados por aglutinación en microplaca con 72 antisueros específicos obtenidos en el Laboratorio de Bacteriología Intestinal del Hospital Infantil de México. La purificación de los antisueros se desarrolló por el método de Edwards y Fife. ${ }^{10}$

Amplificación azarosa del polimorfismo de ADN por la reacción en cadena de la polimerasa ( $P C R$, por sus siglas en inglés) (AAPD-PCR). El ADN total de los 15 aislamientos fue amplificado con el oligonucleótido REP2-Dt, según lo descrito por Eisen D y colaboradores. ${ }^{11}$

Isoelectroenfoque (IEF) y bioensayo. El patrón de bandas de las $\beta$-lactamasas fue obtenido de acuerdo a lo descrito por Matthew y colaboradores. ${ }^{12}$ La identificación de las BLEE se determinó mediante el bioensayo, según Silva y Aguilar. ${ }^{13}$

Electroforesis en gel por campos pulsados (EGCP). Se empleó el método descrito por Kaufmann y colaboradores, ${ }^{14}$ digerido el ADN con la enzima XbaI (Gibco, BRL, Gaithersburg, Md.); los fragmentos obtenidos se separaron en un sistema CHEF-DRIII (Bio-Rad, Hercules, California, Estados Unidos de américa [EUA]). El patrón de bandas fue clasificado de acuerdo con los criterios de Tenover y colaboradores. ${ }^{15}$ 


\section{Cuadro I \\ Datos epidemiológicos Y moleculares de los aislamientos de KLeBsiella PN Eum O Niae, del Hospital Infantil de México, Ciudad de México, junio de 1996}

\begin{tabular}{|c|c|c|c|c|c|c|c|c|c|c|c|c|c|}
\hline \multirow[b]{2}{*}{ No de cepa } & \multirow[b]{2}{*}{ Origen* } & \multicolumn{8}{|c|}{$\mathrm{CMI}(\mu \mathrm{g} / \mathrm{ml})^{\S}$} & \multicolumn{4}{|c|}{ Patrón\# } \\
\hline & & Sala ${ }^{\ddagger}$ & $\overline{C T X}$ & $\begin{array}{c}\text { CTX } \\
+ \\
\text { CLAV }\end{array}$ & CAZ & $\begin{array}{c}\text { CAZ } \\
+ \\
\text { CLAV }\end{array}$ & PM & AZT & Serotipo\& & AAPD & EGC & $\begin{array}{l}\text { Plásmidos } \\
\text { kb }\end{array}$ & $\begin{array}{c}\beta \text {-lactamasa } \\
(\mathrm{pl})\end{array}$ \\
\hline $1331 \mathrm{~N}$ & Posmortem & UCIN & 64 & 0.06 & $>128$ & 4 & 128 & 128 & 61 & I & A & $110,60,54$ & $5.4,7.6,[8.2]$ \\
\hline $1330 \mathrm{~N}$ & Posmortem & UCIN & 64 & 0.06 & $>128$ & 4 & 16 & 128 & 61 & II & A1 & $110,60,<54$ & $5.4,7.6,[8.2]$ \\
\hline $1332 \mathrm{~N}$ & Posmortem & UCIN & 64 & 0.06 & $>128$ & 4 & 128 & 128 & 61 & 1 & A3 & $110,60,<54$ & $5.4,7.6,[8.2]$ \\
\hline$\overline{1327 N}$ & Hemocultivo & UCIN & 64 & 0.06 & $>128$ & 0.25 & 32 & 64 & 61 & I & A3 & 110,54 & $5.4,7.6,[8.2]$ \\
\hline $1328 \mathrm{~N}$ & Hemocultivo & UCIN & 32 & 0.06 & $>128$ & 2 & 8 & 128 & 61 & 1 & A & 110,60 & $5.4,7.6,[8.2]$ \\
\hline $1329 \mathrm{~N}$ & Hemocultivo & UCIN & 0.06 & 0.06 & 16 & 2 & 0.5 & 0.25 & 61 & I & A & $110,<54$ & $5.4,7.6$ \\
\hline $1326 \mathrm{~N}$ & Hemocultivo & UCIN & 2 & 0.06 & 64 & 0.25 & 0.25 & 0.25 & 47 & IV & B & $<54$ & negativo \\
\hline $1333 \mathrm{~N}$ & Posmortem & UCIN & 8 & 0.06 & $>128$ & 4 & 2 & 64 & 64 & II & $D$ & $110,60,<54$ & $5.4,7.6,[8.2]$ \\
\hline $1334 \mathrm{P}$ & Coprocultivo & personal & 4 & 0.06 & 64 & 0.5 & 2 & 64 & 61 & I & A & $110,60,54$ & $5.4,7.6,[8.2]$ \\
\hline $1335 \mathrm{P}$ & Coprocultivo & personal & 64 & 0.06 & $>128$ & 4 & 16 & 128 & 61 & 1 & $\mathrm{~A} 2$ & 110,60 , & $5.4,7.6,[8.2]$ \\
\hline $1338 \mathrm{P}$ & Coprocultivo & personal & 16 & 0.06 & $>128$ & 2 & 2 & 32 & 61 & I & A & $60,<54$ & $5.4,7.6,[8.2]$ \\
\hline 1339P & Coprocultivo & personal & 64 & 0.25 & $>128$ & 2 & 64 & 128 & 61 & 1 & A & $110,<54$ & $5.4,7.6,[8.2]$ \\
\hline 1336P & Coprocultivo & personal & 32 & 0.5 & 128 & 4 & 32 & 128 & 54 & 1 & A1 & $110,<54$ & $5.4,7.6,[8.2]$ \\
\hline $1337 \mathrm{P}$ & Coprocultivo & personal & 4 & 0.06 & 16 & 0.25 & 0.25 & 0.06 & 61 & 1 & $\mathrm{~A} 2$ & $110,60,<54$ & $5.4,7.6$ \\
\hline $1340 \mathrm{P}$ & Coprocultivo & personal & 2 & 0.06 & 16 & 0.125 & 0.5 & 0.06 & $\mathrm{Nt}$ & III & $C$ & $<54$ & $5.4,7.6$ \\
\hline
\end{tabular}

* Cultivos de pacientes fallecidos

₹ UCIN : unidad de cuidados intensivos neonatal

§ CMI: concentración mínima inhibitoria, el resultado es la moda de tres experimentos realizados independientemente CTX: cefotaxima CLAV: ácido clavulánico CAZ: ceftazidima PM: cefpirom AZT: aztreonam

\# AAPD: amplificación azarosa de polimorfismo de ADN, EGCP: electroforesis en gel de campos pulsados, kb: kilobases, pl: punto isoeléctrico, [ ]: $\beta$ lactamasa de espectro extendido

\& $\mathrm{N}$ t: no tipificado

Amplificación por la PCR de bla ${ }_{S H V}$ y secuenciación nucleotídica. Para amplificar el gene $b a_{\mathrm{SHV}}$ se utilizaron los oligonucleótidos SE5 y SB3 descritos por Silva y colaboradores, 2001. ${ }^{5}$ Los productos de la PCR fueron secuenciados con el equipo ABI PRISM 377-18, con el Kit Taq FS Dye terminator Cycle Sequencing. La conversión de la secuencia de nucleótidos a aminoácidos se realizó con el programa Translate tool (http://www. expasy.ch/tools/dna.html). Los alineamientos múltiples de los nucleótidos y aminoácidos se efectuaron en el programa Clustal W del paquete GCG (Genetics Computer Group) usando como secuencia de referencia $b l a_{\mathrm{SHV}-1}{ }^{16}$ (número de acceso a Genbank AFI48850).

\section{Resultados}

Cepas bacterianas. Los 15 aislamientos de K. pneumoniae tuvieron un fenotipo de resistencia a aminoglucósidos y cuando menos a una cefalosporina de tercera gene- ración. Ocho aislamientos correspondieron a pacientes con infección nosocomial y siete de coprocultivos obtenidos del personal paramédico (enfermeras y médicos) que permaneció en contacto con los pacientes. La letalidad en estos pacientes fue de $50 \%$ (4 de 8 pacientes con aislamiento positivo de K. pneumoniae productor de BLEE) (cuadro I).

Pruebas de susceptibilidad antimicrobiana. Los aislamientos analizados fueron susceptibles a cefoxitin, ciprofloxacina e imipenem y resistentes a amikacina $y$, cuando menos, a una cefalosporina de tercera generación, excepto los aislamientos 1329N, 1326N, 1337N y 1340P, (este último obtenido del personal). Este fenotipo sugería que las cepas fuesen productoras de BLEE. Al determinar la CMI para cefotaxima y ceftazidima en combinación con ácido clavulánico (inhibidor de $\beta$-lactamasas de clase A), la CMI disminuyó de 8 a 10 veces, confirmando la producción de BLEE en los ais- 
lamientos analizados de acuerdo con lo indicado por el National Committee for Clinical Laboratory Standars (NCCLS) $)^{15}$ (cuadro I).

Análisis molecular de los aislamientos por serotipificación, amplificación azarosa del polimorfismo del $A D N(A A P D)$ y electroforesis en gel de campos pulsados (EGCP). Se identificaron cuatro serotipos, el mayoritario correspondió al serotipo 61 que incluyó 11 cepas, de las cuales seis pertenecieron a pacientes y cinco al personal. Los serotipos 64, 54 y 47 incluyeron un aislamiento cada uno; el aislamiento 1340P no fue posible tipificarlo (cuadro I). El segundo criterio fue el AAPD que incluyó cuatro grupos, el mayoritario fue el I que comprendió a cinco aislamientos clínicos y a seis del personal; el grupo II incluyó dos aislamientos clínicos y los grupos III y IV incluyeron un aislamiento de personal y uno clínico, respectivamente (cuadro I). El tercer criterio empleado (EGCP) estuvo conformado por una clona mayoritaria $\mathrm{A}$ con tres subtipos (A1 a A3), que incluyeron seis aislamientos clínicos y seis aislamientos del personal, además de tres clonas no relacionadas que correspondieron dos a pacientes $(1326 \mathrm{~N}$ y $1333 \mathrm{~N}$ ) y uno a personal médico (1340C), (cuadro I y figura 1).

Perfil de plásmidos y transferencia de la resistencia. Los aislamientos presentaron de 1 a 3 plásmidos con pesos

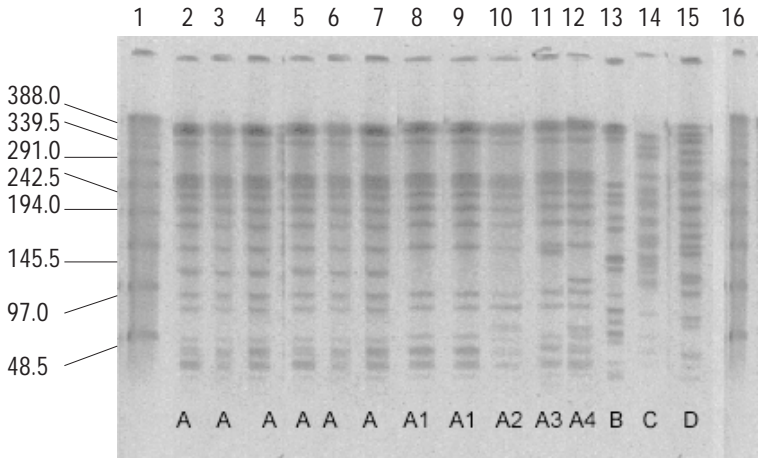

Figura 1. Gel de agarosa representativo de los paTRONES GENERADOS POR ELECTROFORESIS EN GEL DE CAMPOS PULSADOS DE ADN CROMOSOMAL DIGERIDO CON X ba I. Hospital Infantil de México, Ciudad de MéXICO, 1996*

* Carriles 1 y 16 marcador peso molecular del ADN del fago Lambda, Aislamientos de K. pneumoniae productores de $\beta$-lactamasas de espectro extendido 2) $1331 \mathrm{~N}$; 3) $1329 \mathrm{~N}$; 4) 1334P; 5) $1338 \mathrm{P}$; 6) $1328 \mathrm{~N}$; 7 ) $1339 \mathrm{P}$; 8$) 1330 \mathrm{~N}$; 9) 1336P; 10) 1337P; 11) 1327N ; 12) 1332N ; 13) 1326N ; 14) $1333 \mathrm{~N}$; 15) $1340 \mathrm{P}$. moleculares de $<54,60$ y $110 \mathrm{~kb}$, aunque en ningún caso fue posible transferir, por conjugación bacteriana, la resistencia a cefotaxima o ampicilina.

IEF de $\beta$-lactamasas y detección de actividad de cefotaximasa. Once de los quince aislamientos expresaron $\beta$-lactamasas con punto isoecléctrico (pI) de 5.4, 7.6 y 8.2 (seis aislamientos clínicos y cinco de personal); esta última enzima tuvo la actividad de cefotaximasa (cuadro I). Los aislamientos susceptibles a $\beta$-lactámicos correspondieron a una cepa de origen clínico (1329N) y dos al personal (1337P y 1340P), las cuales expresaron $\beta$-lactamasas con pI de 5.4 y 7.6. El aislamiento $1326 \mathrm{~N}$ no fue productor de $\beta$-lactamasas.

Caracterización molecular de las $\beta$-lactamasas: amplificación y secuencia nucleotídica. Dos aislamientos, $1333 \mathrm{~N}$ y 1335P, fueron seleccionados al azar como representantes de las dos poblaciones de aislamientos clínicos y del personal. Los resultados de biología molecular indicaron que la $\beta$-lactamasa con $\mathrm{pI}$ de 5.4 correspondió a la familia TEM, la de pI de 7.6 a SHV-1, y la de pI de 8.2 a la variante SHV-5 (BLEE).

\section{Discusión}

El desarrollo de las técnicas de tipificación clonal ha permitido detectar el origen y la ruta de epidemias hospitalarias, así como definir estrategias de control y eliminación de la infección. ${ }^{17}$ Los tres criterios que se emplearon para determinar la relación clonal entre los aislamientos mostraron una correlación muy estrecha; de esta forma se pudo identificar a una clona con tres subtipos como la responsable de la infección causada en los pacientes y la colonización en el personal médico indicando una posible diseminación en forma cruzada, como ya se ha descrito en especies de Staphylococcus y enterobacterias como E. coli, Serratia marcsecens y K. pneumoniae como los principales agentes etiológicos de la infección. ${ }^{18,19}$

La mayoría de los aislamientos expresaron dos tipos de enzimas, la $\beta$-lactamasa TEM-1 y dos del tipo SHV. Por otra parte, estos datos correlacionan con el patrón electroforético de las $\beta$-lactamasas, donde la segunda enzima ( $\mathrm{pI}$ de 8.2) tiene solamente la actividad de cefotaximasa en el bioensayo.

En relación con la susceptibilidad con los diferentes antibióticos $\beta$-lactámicos, los valores de CMI para cefotaxima y cefepime presentaron cierta variabilidad que puede explicarse mediante la posible adquisición de otros mecanismos de resistencia a antibióticos $\beta$-lactámicos, tales como la alteración en la permeabi- 
lidad mediado por "porinas" o la adquisición de bombas de expulsión. ${ }^{20}$

Aunque la mayoría de los aislamientos estudiados contienen al menos un plásmido de 110, 60 o $<54$ $\mathrm{kb}$, sin embargo, no se logró la transferencia por conjugación bacteriana.

Se ha descrito a K. pneumoniae como uno de los principales agentes causantes de bacteriemias nosocomiales. ${ }^{1,4,5}$ En este estudio se describen siete casos de bacteriemias secundarias y aunque no se tienen datos sobre los sitios de infección primaria involucrados en la generación de las bacteriemias, se sugiere que las infecciones endovasculares podrían asociarse con los casos estudiados, debido a una posible contaminación de soluciones parenterales y descuido de las medidas asépticas empleadas en la inserción y mantenimiento de catéteres vasculares. Estas serían las principales causas de la vía de entrada de este agente bacteriano y posiblemente los responsables de una diseminación vertical clonal. ${ }^{21,22}$

Los resultados de este estudio, aun cuando no son recientes, ponen de manifiesto que en la sala de la UCIN de esta institución debe practicarse permanentemente una serie de medidas tales como: establecer el número de pacientes críticos atendidos por el personal de enfermería, crear programas continuos de técnicas de lavado de manos, establecer protocolos que permitan detectar la concentración bactericida de los antisépticos empleados en los procedimientos relacionados con pacientes y promover el uso racional de antibióticos para evitar el surgimiento y diseminación de bacterias multirresistentes.

\section{A gradecimientos}

Se agradece a la Bióloga Z Becerra y a la Técnica T Rojas su excelente asistencia técnica.

\section{Referencias}

1. González-Vértiz A,Alcántar-C uriel D, C uauhtli M, D aza C, Gayosso C, Solache $\mathrm{G}$ et al. Multiresistant extended-spectrum $\beta$-lactamaseproducing Klebsiella pneumoniae causing an outbreak of nosocomial bloodstream infection. Infect Control Hosp Epidemiol 2001; 22:723-725. 2.Vernon MO,Trick W E, W elbel SF, Peterson BJ,W einstein RA. Adherence with hand hygiene: Does number of sinks matter? Infect Control Hosp Epidemiol 2003; 24:224-225.

3. Jacoby G, Busch K. Aminoacid sequences for TEM, SHV and OXA extended spectrum and inhibitor resistant $\beta$-lactamasa. Lahey $C$ linic. D isponible en: http://www.lahey.org/Studies/?D =http://www.lahey.org/ studies/webt.stm\&C = 404, last modified - 09/03/04
4. Martínez G,Alpuche C,A naya C,Alcántara D, G ayosso C, D aza C et al. $O$ utbreak of nosocomial sepsis and pneumonia in a $\mathrm{N}$ ewborn Intensive $\mathrm{C}$ are Unit by multiresistant extended-spectrum $\beta$-Lactamaseproducing Klebsiella pneumoniae: High impact on mortality. Infect Control Hosp Epidemiol 2001; 22:725-728.

5. Silva J, G atica R, A guilar C, Becerra Z, G arza U,Velázquez M et al. 0 utbreak of infection with extended-spectrum- $\beta$-lactamase-producing Klebsiella pneumoniae in a Mexican hospital. J C lin Microbiol 2001; 39:

3193-3196.

6. Miranda G, C astro N , Leaños B, Valenzuela A, Garza-Ramos U, Rojas T et al. Clonal and horizontal dissemination of Klebsiella pneumoniae expressing SHV-5 extended-spectrum $\beta$-Lactamase in a Mexican pediatric hospital. J Clin Microbiol 2004; 42:30-35.

7. N ational Committee for Clinical Laboratory Standards. Methods for dilution antimicrobial susceptibility test for bacteria that grow aerobically. A pproved standard M100-S12. W ayne (PA): N ational Committee for Clinical Laboratory Standards; 2002; 22:42-46. 8. Kieser T. Factors affecting the isolation of CCC DN A from Streptomyces lividans and Escherichia coli. Plasmid 1984; 12:10-36. 9. Miller J. Experiments in molecular genetics. Cold Spring Harbour (N Y): Cold Spring Harbour Laboratory; 1992: 82-85.

10. Edwards PR, Fife MA. C apsule types of Klebsiella. 1952; 91:92-104

11. Eisen D, Russell EG, Tymms M, Roper EJ, Grayson ML,Turnidge J. Random amplified polymorphic DNA and plasmid analysis used in investigation of an outbreak of multiresistant Klebsiella pneumoniae. J Clin Microbiol 1995; 33:713-717.

12. Matthew $G$, Hedges $R$, Smith J. Types of $\beta$-lactamases determined by plasmid in gram-negative bacteria. J Bacteriol 1979; 138:657-662.

13. Silva J, A guilar C. B-Lactamase bioassay:A simplified method to determine extended-spectrum $\beta$-lactamases (ESBL) in enterobacteria. Arch Med Res 1997; 28:285-287.

14. Kaufmann ME. Pulsed-field gel electrophoresis. Methods Mol Med 1998; 15:17-31.

15. Tenover FC, Arbeit RD, Goering RV, Mickelsen PA, Murray BE, Persing $\mathrm{DH}$ et al. Interpreting chromosomal DN A restriction patterns produced by pulsed- field gel electrophoresis: C riteria for bacterial strain typing.J Clin Microbiol 1995; 33:2233-2239.

16. Barthelemy M, Peduzzi J, Labia R. Complete amino acid sequence of p453-plasmid-mediated PIT-2 $\beta$-lactamase (SHV-1).J Biochem 1988; 251:73-79.

17. Mondino SS, C astro AC, Mondino PJ, C arvalho Mda G, Silva KM, Teixeira LM. Phenotypic and genotypic characterization of clinical and intestinal enterococci isolated from inpatients and outpatient:Two Brazilian hospitals. Microb D rug Resist 2003; 9:167-174.

18. Girou E,Azar J,W olkenstein P, Cizeau F, Brun-Buisson C, Roujeau JC. Comparison of systematic versus selective screening for methicillinresistant Staphylococcus aureus carriage in a high-risk dermatology ward. Infect Control Hosp Epidemiol 2000; 21:583-587.

19. Martínez Jl, Baquero F. Interactions among strategies associated with bacterial infection: Pathogenicity, epidemicity, and antibiotic resistance. Clin Microbiol Rev 2002; 15:647-679.

20. Webber M, Piddock L.The importance of efflux pumps in bacterial antibiotic resistance. J Antimicrob Chemother 2003; 51:9-11.

21. Hernández RI, G aitán MJ, G aitán GE. Extrinsic contamination of intravenous infusates administered to hospitalized children in Mexico.J Pediatr Infect D is 2000; 19: 888-890.

22. Macías AE, Bruckner DA, H indler JA. Parenteral infusions as culture media from a viewpoint of nosocomial bacteremia. Rev Invest C lin 2000; 52: $39-43$. 